\title{
Utility of Dual Red Imaging for Endoscopic Hemostasis of Gastric Ulcer Bleeding
}

\author{
Yoko Kubosawa Hideki Mori Ai Fujimoto \\ Department of Gastroenterology and Hepatology, National Hospital Organization Tokyo Medical Center, \\ Tokyo, Japan
}

\section{Keywords}

Dual red imaging · Hemostasis · Gastric ulcer bleeding ·

Red Dichromatic Imaging

\begin{abstract}
Dual red imaging (DRl; Red Dichromatic Imaging (RDI)) is a new type of image-enhanced endoscopy composed of two long wavelengths that together can visualize vessels in the deep submucosa of the gastrointestinal wall. We treated a case of gastric ulcer bleeding in which the bleeding point could be clearly visualized using DRI. A 71-year-old man who was taking warfarin for atrial fibrillation noticed tarry stool, entered a state of hypovolemic shock, and underwent emergency esophagogastroduodenoscopy. The procedure was performed with a GIF-Y0058 prototype endoscope (Olympus (o., Tokyo, Japan) equipped with a DRI mode. An ulcer with active bleeding was found in the upper portion of the stomach, but the massive volume of the bleeding made it difficult to identify the bleeding point using white light imaging (WLI). Upon switching to DRI mode by pushing a button on the endoscope, the bleeding point could be identified rapidly and precisely, as it appeared in a deeper yellow than the surrounding area. Complete hemostasis was thereupon achieved. This case demonstrates that the DRI mode may be useful for detecting bleeding points that are difficult to detect using WLI.

(c) 2019 S. Karger AG, Basel
\end{abstract}

\section{Introduction}

Gastric ulcer bleeding is a common disease that sometimes requires emergency endoscopic hemostasis. In cases of massive hemorrhage, however, it is often difficult to identify the source of the bleeding and achieve hemostasis. Dual red imaging (DRI; Red Dichromatic Imaging (RDI)) is a new type of image-enhanced endoscopy that can identify the bleeding point easily in an active bleeding gastric ulcer.

\section{Case Presentation}

A 71-year-old man who was taking warfarin for atrial fibrillation noticed tarry stool and entered a state of hypovolemic shock. His blood tests showed anemia and elevated blood urea nitrogen. His PT-INR did not prolong; 1.82 . We suspected upper gastrointestinal bleeding. After rapid infusion of $500 \mathrm{~mL}$ lactated Ringer's solution and $280 \mathrm{~mL}$ red blood cell transfusion, the patient's vital signs became stable. We then promptly examined him by esophagogastroduodenoscopy. The procedure was performed with a GIF-Y0058 prototype endoscope (Olympus Co., Tokyo, Japan) equipped with a DRI mode. We found an ulcer with active bleeding from a lesion in the upper portion of the stomach. Massive bleeding from the lesion, however, made it difficult to identify the bleeding point using white light imaging or achieve perform endoscopic hemostasis (Fig. 1a). Upon switching to DRI mode by pressing a button on the endoscope, the bleeding point appeared in a deeper yellow than the sur-

\section{KARGER}

(c) 2019 S. Karger AG, Basel

karger@karger.com

www.karger.com/ddi
Ai Fujimoto, MD, PhD

Department of Gastroenterology and Hepatology

National Hospital Organization Tokyo Medical Center, 2-5-1 Higashigaoka, Meguro-ku Tokyo 152-8902 (Japan)

E-Mail aifujimoto0517@gmail.com 
Fig. 1. a Massive bleeding from the lesion precluded the identification of the bleeding point using white light imaging. b Upon switching to dual red imaging mode, the bleeding point was depicted in a deeper yellow than the surrounding area. The red arrow shows the bleeding point. c Complete hemostasis was achieved promptly. d 10 months later, the ulcer closed completely.

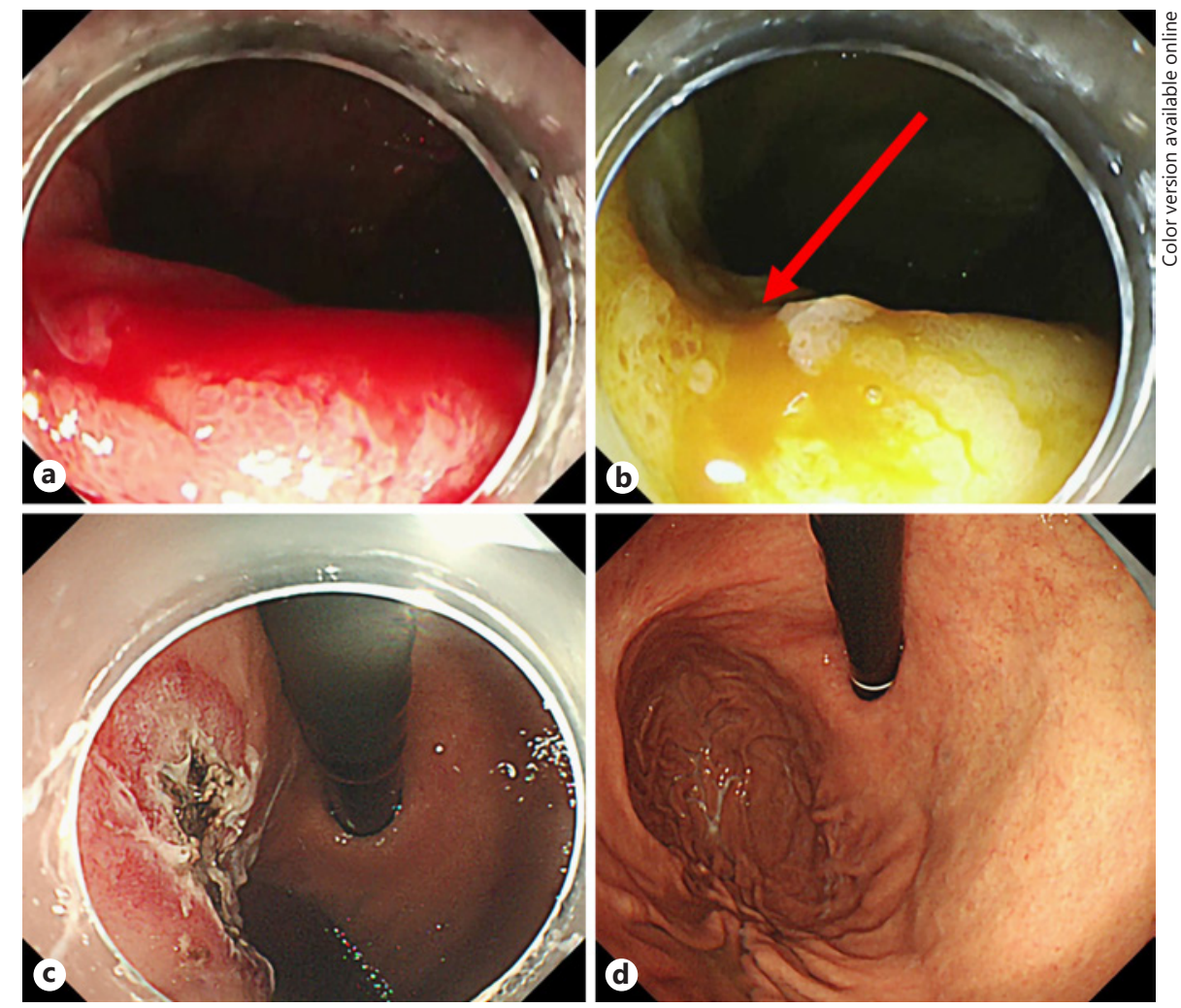

rounding area, enabling us to identify it rapidly and precisely (Fig. 1b). We grasped and coagulated the exposed vessel with hemostatic forceps (Coagrasper; Olympus Co., Tokyo, Japan) by a soft coagulation (effect 5, $80 \mathrm{~W}$ ) using an electrosurgical unit (VIO300D; ERBE, Tübingen, Germany). The procedure achieved prompt hemostasis (Fig. 1c) (Video 1). No re-bleeding occurred after the patient resumed treatment with warfarin. 10 months later, the ulcer closed completely (S2 stage) (Fig. 1d). Our patient had open-type atrophic gastritis, and his serum $\mathrm{He}$ licobacter pylori antibody test was positive. He underwent eradication therapy after ulcer treatment.

\section{Discussion}

DRI is a new image-enhanced endoscopy technique that uses narrow-band light at two long wavelengths: 600 and $630 \mathrm{~nm}$. DRI can detect thick blood vessels at a depth range of 1,000-1,500 $\mu \mathrm{m}$ from the mucosal surface of the gastrointestinal wall [1]. Naganuma et al. [2] reported that DRI was useful to assess the severity of inflammation using the deep vascular pattern in patients with ulcerative colitis. Ninomiya et al. [3] proposed that DRI may enhance both the safety and speed of colorectal endoscopic submucosal dissection by improving the visibility of the arteries. Furuichi et al. [4] similarly found that DRI increased the visibility of the esophageal varices and red color sign.

DRI has also been reported to be useful for visually identifying the bleeding point and achieving hemostasis in both duodenal ulcer bleeding and bleeding during endoscopic submucosal dissection $[5,6]$. The hemoglobin concentration in the blood of a patient differs between the thick blood flow at the bleeding point and the blood diluted by the water jet in the surrounding area. Due to the light absorption characteristics of blood hemoglobin, the light at the narrow-band $600 \mathrm{~nm}$ wavelength improves the visibility of the bleeding point by clearly visualizing this difference in hemoglobin concentration. The high visibility of the bleeding point under a water jet flow is therefore an advantage, even though the bleeding point is on the gravity side.

This report is the first to demonstrate the usefulness of DRI for attaining hemostasis in a case with bleeding from a gastric ulcer in which the bleeding point was difficult to identify by white light imaging. DRI has the potential to simplify hemostatic treatment for gastric ulcer bleeding, a problem frequently experienced in daily clinical practice. 


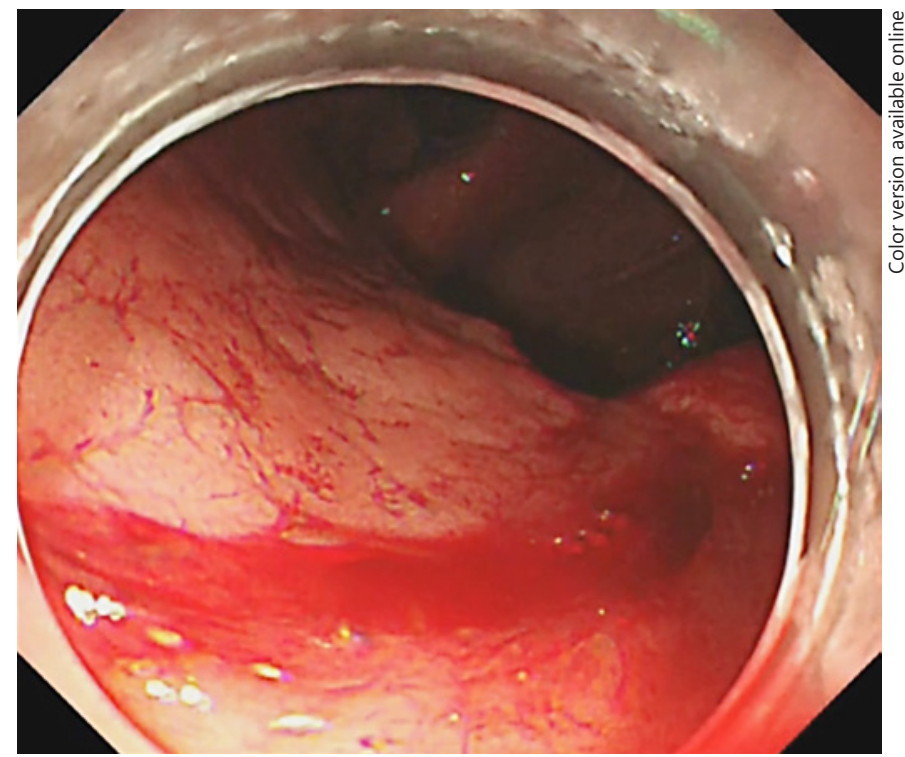

Video 1. We examined the patient by esophagogastroduodenoscopy suspecting upper gastrointestinal bleeding. We found a gastric ulcer with active bleeding in the upper portion of the stomach. Massive bleeding precluded the identification of the bleeding point using white light imaging. Upon switching to dual red imaging mode, the bleeding point was depicted in a deeper yellow than the surrounding area, enabling us to identify it rapidly and precisely. We coagulated the exposed vessel with hemostatic forceps by a soft coagulation using an electrosurgical unit. The procedure achieved prompt hemostasis.

\section{Acknowledgement}

The authors would like to thank to Dr. Makoto Igarashi for his technical support and helpful assistance.

\section{Statement of Ethics}

The authors received the patient's written informed consent. The study protocol was approved by the National Hospital Organization Tokyo Medical Center Review Board (R18-043).

\section{Disclosure Statement}

The prototype endoscope (GIF-Y0058) was provided free of charge by Olympus Co.

\section{Funding Sources}

The authors have not declared a specific grant for this research from any funding agency.

\section{Author Contributions}

Y.K. performed hemostasis with the patient's consent for treatment. H.M. and A.F. provided assistance and advice on hemostasis treatment. All of the authors read and approved the final manuscript.

\section{References}

1 Yahagi N, Fujimoto A, Horii J, Uraoka T, Shimoda $M$, Takabayashi K, et al. Dual red imaging: a novel endoscopic imaging technology visualizing thick blood vessels in the gastrointestinal wall. Endosc Int Open. 2019; 7(12):E1632-5.

2 Naganuma M, Yahagi N, Bessho R, Ohno K, Arai M, Mutaguchi M, et al. Evaluation of the severity of ulcerative colitis using endoscopic dual red imaging targeting deep vessels. Endosc Int Open. 2017 Jan;5(1):E76-82.
3 Ninomiya Y, Oka S, Tanaka S, Hirano D, Sumimoto K, Tamaru Y, et al. Clinical impact of dual red imaging in colorectal endoscopic submucosal dissection: a pilot study. Therap Adv Gastroenterol. 2016 Sep;9(5):679-83.

4 Furuichi Y, Gotoda T, Moriyasu F, Ogawa S, Kasai $Y$, Takeuchi H, et al. Dual red imaging (novel advanced endoscopy) can increase visibility and can predict the depth in diagnosing esophageal varices. J Gastroenterol. 2017 May;52(5):568-76.
5 Tanaka H, Oka S, Tanaka S. Endoscopic hemostasis for spurting duodenal bleeding using dual red imaging. Dig Endosc. 2017 Nov; 29(7):816-7.

6 Fujimoto A, Saito Y, Abe S, Hoteya S, Nomura $\mathrm{K}$, Yasuda $\mathrm{H}$, et al. Haemostasis treatment using dual red imaging during endoscopic submucosal dissection: a multicentre, openlabel, randomised controlled trial. BMJ Open Gastroenterol. 2019 Mar;6(1):e000275. 